\title{
Complicaciones del uso del pesario con soporte y sin soporte en el prolapso genital completo
}

\author{
Franklin J. Espitia-De La Hoz*
}

\begin{abstract}
*Médico especialista en Ginecología y Obstetricia. Universidad Militar Nueva Granada. Uroginecología y reconstrucción del piso pélvico. Fundación Universitaria de Ciencias de la Salud. Bogotá. Universidad Estatal de Campinas. Campinas. Brasil. Unidad de Ginecología Urológica. Clínica La Sagrada Familia. Armenia. Quindío. Colombia.
\end{abstract}

Correspondencia: Dr. Franklin Espitia. Dirección: Carrera 12 \# 0 - 75 Consultorio 508, Clínica El Café. Armenia. Quindío. Colombia. Correo electrónico: espitiafranklin@hotmail.com

\section{RESUMEN}

Introducción: La prevalencia del prolapso genital se ha incrementado; a menudo cursa de forma asintomática, se suele presentar con síntomas de un "bulto" vaginal. Las opciones de tratamiento incluyen ejercicios del suelo pélvico, manejo expectante, el uso de dispositivos mecánicos y corrección quirúrgica. Objetivo: Evaluar las complicaciones inmediatas y tardías del uso del pesario sin soporte y con soporte, en el manejo conservador del prolapso genital completo. Materiales y métodos: Estudio descriptivo, observacional, transversal y comparativo. Se revisaron 108 historias clínicas de las pacientes atendidas por prolapso genital completo y se evaluaron a las que se les manejó de forma conservadora con pesarios con soporte (Grupo A: 9) y sin soporte (Grupo B: 18). Los dos grupos se compararon tomando en cuenta el aumento de secreción vaginal, aparición de erosión y úlceras vaginales, impactación, fístulas, atipias citológicas, incarceración, hidronefrosis, infección vaginal y complicaciones intestinales. Resultados: Se analizaron 27 pacientes en total, 9 manejadas con pesarios con soporte y 18 con pesarios sin soporte. Hubo diferencia significativa en cuanto al aumento de secreción vaginal con menor secreción en uso del pesarios sin soporte $(p=0,045)$. Se encontró diferencia en la aparición de erosión y úlceras vaginales ( $p<$ 0,05). Conclusiones: Hay diferencia significativa en cuanto al aumento de la secreción vaginal y la aparición de erosión y úlceras vaginales en el uso de pesarios sin soporte en comparación con el uso de pesarios con soporte. MÉD UIS. 2015;28(3):309-15.

Palabras clave: Pesarios. Indicaciones. Tratamientos Conservadores del Órgano. Prolapso de Órgano Pélvico. Prolapso Uterino.

\section{Complications from the use of pessary with support and without support in the complete genital prolapse}

\section{ABSTRACT}

Introduction: The prevalence of genital prolapse has increased; often it is asymptomatic, usually presents with symptoms of a vaginal "bulge". Treatment options include pelvic floor exercises, expectant management, the use of mechanical devices and surgical correction. Objectives: Evaluate the immediate and late complications of the use of pessary without support and with support in the conservative management of the entire genital prolapse. Materials and Methods: Descriptive, observational, transversal and comparative study. 108 medical records of patients treated by complete genital prolapse were reviewed and evaluated which were managed conservatively with pessaries supported (Group A: 9) and unsupported (Group B: 18). The two groups are compared taking into account the increased vaginal discharge, vaginal appearance of erosion and ulcers, impaction, fistulas, cytologic atypia, incarceration, hydronephrosis, vaginal infection and intestinal complications. Results: 27 patients were analyzed in total, 9 handled pessaries supported and 18 unsupported pessaries. There was significant difference in terms of vaginal discharge increasment with less secretion in unsupported use of pessaries ( $p=0.045)$. A difference was found in the occurrence of erosion and vaginal ulcers $(p<0.05)$. Conclusions: There is a significant difference in terms of vaginal discharge increasment and occurrence of erosion and ulcers vaginal pessaries using unsupported compared with using supported pessaries. MÉD UIS. 2015;28(3):309-15.

Keywords: Pessaries. Indications. Organ Sparing Treatments. Pelvic Organ Prolapse. Uterine Prolapse.

Artículo recibido el 05 de mayo de 2015 y aceptado para publicación el 10 octubre de 2015. 
¿Cómo citar este artículo?: Espitia-De La Hoz FJ. Complicaciones del uso del pesario con soporte y sin soporte en el prolapso genital completo. MÉD UIS. 2015;28(3):309-15.

\section{INTRODUCCIÓN}

El prolapso genital es el descenso de uno o más de los órganos pélvicos: vejiga (pared anterior), útero (ápice), cúpula vaginal, recto (pared posterior), a través de la vagina y en dirección al periné $\mathrm{o}$ canal anal, que se produce como consecuencia del debilitamiento de sus elementos de suspensión y sustentación ${ }^{2-4}$; y que se caracteriza por ser una patología frecuente dentro de la consulta de uroginecología. En un estudio colombiano en el que participaron 13824 pacientes, el 26,6\% estaban histerectomizadas y se les diagnosticó prolapso genital en el $68,38 \%$; y el $73,4 \%$ no histerectomizadas, a las que se les diagnosticó prolapso genital en el 31,62\%. En el estudio Women's Health Initiative (WHI) de la totalidad de mujeres incluidas en el seguimiento, el $40 \%$ manifestó algún grado de prolapso genital y se evidenció un $14 \%$ de prolapso apical ${ }^{6}$. En estudios de EEUU se resalta que existe un riesgo quirúrgico por prolapso o incontinencia urinaria del $11,1 \%$ a los 80 años, y de un $13 \% 7$ al $29,2 \%^{8}$ de las pacientes sometidas a cirugía del prolapso requieren una segunda cirugía, otros reportan $30,8 \%$ de prolapso, pero sólo el $2 \%$ se localizaban por debajo del introito vaginal9.

El prolapso genital aparece como la primera indicación de histerectomía en mujeres postmenopáusicas (entre el $15 \%$ al $18 \%$ ); y el $20 \%$ de las mujeres en espera para una cirugía mayor ginecológica, lo son por esta condición ${ }^{10}$. Sin embargo, las tasas de recurrencias quirúrgicas no son despreciables: $22 \%$ en el compartimento anterior, $30 \%$ en el compartimento apical y $24 \%$ en el compartimento posterior ${ }^{11,12}$. Se estima que el $33 \%$ de las mujeres adultas sufren alguna forma de disfunción del suelo pélvico, y que al $33,5 \%$ de ellas se le hará una intervención quirúrgica relacionada con esta condición. Se calcula que en la población femenina solo un $3 \%$ de las pacientes presenta prolapso genital severo (III y IV grado), y es probable que entre el $3 \%$ a $6 \%$ de la población general desarrolle un prolapso de esas características en algún momento de su vida ${ }^{13,14}$.

Entre los factores de riesgo de prolapso genital, los más comunes son: edad mayor de 65 años, multiparidad, sobrepeso, constipación crónica, levantar cargas pesadas en forma repetitiva ${ }^{15}$, episiotomía rutinaria, segunda etapa del parto prolongada, laceración del esfínter anal, uso de fórceps ${ }^{16}$, neumopatías crónicas (EPOC), tabaquismo ${ }^{17}$, menopausia, trauma, factores genéticos, raza, enfermedades musculo esqueléticas, neuromusculares (espina bífida) y del tejido conectivo (Ehlers-Danlos, síndrome de Marfán) ${ }^{18}$. Las manifestaciones clínicas se caracterizan por ver o sentir un "bulto" vaginal, o la sensación de abultamiento o protrusión vaginal ${ }^{19}$; también pueden presentarse síntomas urinarios como incontinencia urinaria, urgencia urinaria, retención urinaria o disfunción miccional, disfunción defecatoria caracterizada por esfuerzo defecatorio, evacuación incompleta, inmovilización de la pared vaginal posterior o digitación del recto durante la defecación o incontinencia fecal ${ }^{20}$.

Existen diferentes sistemas de clasificación del prolapso de órganos pélvicos, pero las dos más comunes son el de Baden-Walker ${ }^{21}$ y el Pelvis Organ Prolapse Quantitation System (POP-Q) ${ }^{22}$, basado en demostrar la máxima extensión del prolapso en el examen físico, y fue el sistema de clasificación empleado en este estudio ya que es el aprobado por la International Continence Society ${ }^{23}$, American Urogynecologic Society y Society of Gynecologic Surgeons ${ }^{24}$.

El tratamiento convencional y tradicionalmente utilizado en el manejo del prolapso genital completo, ha sido la histerectomía vaginal con colporrafia anterior y posterior, sin embargo, actualmente las mujeres insisten en conservar su útero aunque carezcan de interés reproductivo, es por ello que se debe tener en cuenta el deseo gestacional de la paciente antes de considerar una alternativa quirúrgica como la histerectomía, aun cuando pueda existir una recidiva o progresión tras el parto. Después de este procedimiento, no necesariamente deben ser realizados los partos por cesárea ${ }^{25,26}$. El tratamiento del prolapso genital completo, hoy en día incluye la expectación con tratamiento médico conservador, el uso de pesarios, la fisioterapia y la cirugía (obliterativa o reconstructiva) en última instancia ${ }^{27-9}$.

El uso de pesarios ha sido considerado para ser utilizado mujeres con prolapso sintomático que no aceptan la cirugía y optan por un tratamiento 
conservador, en pacientes con problemas quirúrgicos o por médicos que los prefieren a la cirugía, en jóvenes que tienen interés reproductivo y si existe necesidad temporal para conseguir o continuar un embarazo ${ }^{30-3}$. Los pesarios intravaginales son una opción de tratamiento en el manejo de los prolapsos genitales ${ }^{34}$, y se pueden indicar como primera línea terapéutica en el manejo del prolapso genital completo ${ }^{35,36}$, en mujeres de gran riesgo quirúrgico o mayores con un grado de satisfacción alto ${ }^{37-9}$, se les considera una medida conservadora a tener en cuenta, ya que son mínimamente invasivos, de bajo costo, seguros y efectivos ${ }^{40}$; aunque en la actualidad habían sido relegados a pacientes con contraindicación quirúrgica o deseo de embarazo ${ }^{41}$.

Se estima que las pacientes que usaron el pesario como método de tratamiento del prolapso genital, se muestran satisfechas y continúan con su uso durante largo tiempo ${ }^{42,43}$. Se reportó que al año del uso del pesario se observó una mejoría del grado del prolapso, lo que podría relacionarse con algún efecto terapéutico asociado a su uso continuo ${ }^{44-6}$. Entre las potenciales complicaciones relacionadas con el uso del pesario sobresalen: atipias citológicas inflamatorias, aumento de la secreción vaginal, cáncer vaginal47 (dada la inflamación crónica junto con la infección por VPH), complicaciones intestinales, incarceración cervical del pesario, erosión y úlceras vaginales, fístulas: vesicovaginal ${ }^{48}$, rectovaginal ${ }^{49}$, hidronefrosis, impactación en vagina ${ }^{50}$, infección vaginal, peritonitis, entre otras ${ }^{51}$. Por lo anterior, el objetivo de este artículo es evaluar las complicaciones inmediatas y tardías del uso del pesario sin soporte y con soporte, en el manejo conservador del prolapso genital completo.

\section{MateRIALES y Métodos}

\section{DISEÑO}

Estudio transversal, descriptivo y comparativo, llevado a cabo en el Servicio de Uroginecología de la Clínica la Sagrada Familia, Armenia, Quindío, Colombia. Las indicaciones del uso del pesario fueron: gran riesgo quirúrgico por cardiopatías y neumopatías; en mujeres previamente operadas con recurrencia del prolapso anterior, en espera de la cirugía y no aceptación de la cirugía. Los criterios de exclusión fueron mujeres con infecciones vaginales o cervicales, neoplasias vaginales o cervicales e incapacidad para la inserción del pesario.
Se revisaron las 108 historias clínicas, notas médicas, notas de evolución y censos del seguimiento, de las pacientes atendidas por prolapso genital completo, y se evaluaron a las que se les manejó de forma conservadora con pesarios con soporte (Grupo A: 9) y sin soporte (Grupo B: 18), en la búsqueda de complicaciones relacionadas con el uso del pesario: flujo vaginal, sangrado genital, dolor o sensación de expulsión del pesario, erosión y úlceras vaginales, atendidas desde el 1 de enero de 2009 hasta el 30 de junio de 2012 por prolapso genital completo, a las que se les formuló el uso de pesario sin soporte y con soporte. Las pacientes fueron atendidas por el mismo especialista en todos los casos, el que se encargó de educar y entrenar a las pacientes en el adecuado uso del pesario a fin de que pudieran insertarlo y retirarlo por sí mismas, se les enseñó a introducirse el pesario por la mañana a través de la vagina y volver a retirarlo por la noche, durante la primera semana, luego semanalmente y finalmente cada mes. A las mujeres se les insertaba el pesario, y después se les comprobaba si el pesario quedaba colocado correctamente; para ello se le presionaba el abdomen inferior, se le pedía toser, que saltara y deambulara. A continuación, el médico volvía a comprobar la inserción del pesario.

A todas las pacientes se le eligió el pesario del menor tamaño que le ofreciera suficiente sujeción y se les realizó toma de examen Papanicolau (citología cervical), uroanálisis y se les manejó con profilaxis de óvulos con clotrimazol 100 mg más clindamicina $100 \mathrm{mg}$, durante tres días previos al inicio del pesario.

\section{ANÁLISIS ESTADÍSTICO}

Los datos recolectados fueron llevados a un listado de datos en Microsoft Excel. La estadística descriptiva se expresan en medias, rangos y desviaciones estándar ( \pm ) para datos continuos, frecuencia absoluta y porcentajes con intervalos de confianza del 95\% para datos categóricos; las diferencias fueron evaluadas con la prueba $t$ de Student para la comparación de medias, y el test de la chi cuadrado $\left(x^{2}\right)$ de Pearson para comparar variables cualitativas con un valor significativo para una $p<$ 0,05 .

El proyecto de investigación del estudio fue estudiado y aprobado por las Comisiones de Ética e Investigación de la clínica. 


\section{Resultados}

Se incluyó en el estudio un total de 27 mujeres con prolapso genital completo a las que se les dio manejo conservador mediante el uso de pesarios con soporte (Grupo A) y sin soporte (Grupo B).

La edad de las pacientes fluctuó entre 39 y 75 años, con una media de 57 años. El índice de masa corporal osciló entre 24 y 36, con una media de 27. La paridad fluctuó entre dos y cinco, con una media de tres. El tiempo de uso fue de seis meses a tres años, con una media de 18 meses. Los antecedentes médicos arrojan: Hipertensión Arterial, en 12 casos; Enfermedad Pulmonar Obstructuva Crónica, en tres casos; diabetes, en nueve casos; cardiopatías en nueve casos y osteoartrosis en 12 casos. Los antecedentes quirúrgicos en 12 casos de colporrafia anterior o posterior. El número de pesario más utilizado fue el número 6 en 18 casos y en ningún caso fue necesario ajustar el pesario para un número mayor o menor. Se suspendió temporalmente el uso del pesario, durante un tiempo de uno a tres meses, hasta que sanaron las lesiones ulcerosas en las ochos pacientes en que se presentó esta complicación (cuatro con pesarios sin soporte y cuatro con pesarios con soporte).

En el grupo de las 27 pacientes estudiadas, 9 se manejaron con pesarios con soporte y 18 con pesarios sin soporte. De todas las pacientes atendidas 11 $(40,7 \%)$ fueron no caucásicas y 16 (59,2\%) fueron caucásicas, demostrando una vez más que la raza es un factor predisponente en la aparición del prolapso genital. Las caucásicas tuvieron una edad media de $47,1 \pm 18,9$ años y las no caucásicas una edad media de $61,7 \pm 11,3$ años. Sí hubo diferencia significativa de las edades medias entre no caucásicas y caucásicas, corroborada mediante la prueba $t$ de Student $(p<$ $0,05)$. Las caucásicas presentaron el prolapso genital completo a edades más tempranas.

De las mujeres con prolapso genital completo a las que se dio manejo mediante el uso de pesario con soporte, dos $(22,2 \%)$ fueron no caucásicas y siete $(77,7 \%)$ caucásicas. La media de edad en este grupo fue de 56,2 años. Al realizar el análisis por raza se encontró que a las que se les manejó con el uso de pesario sin soporte nueve (50\%) fueron no caucásicas y nueve (50\%) caucásicas. La media de edad de estas pacientes fue de 55,5 años.

Se aplicó la prueba chi cuadrado para determinar si había relación entre el tipo de uso de pesario y la raza, no existiendo un nivel de significancia aceptable $(p=$ $0,166)$, es decir, no se presentó relación entre el uso de algún tipo de pesario y la raza, también se aplicó la prueba $t$ de Student para determinar diferencia entre las medias de la edad de ambos grupos, uso del pesario con soporte y uso del pesario sin soporte, determinando que no hay diferencia entre las edades en ambos grupos $(p=0,92)$.

En cuanto al aumento de la secreción vaginal, se refiere una media de $8 \pm 1,4$ de quejas en el grupo tratado con pesario con soporte, mientras en el grupo tratado con pesario sin soporte la media del

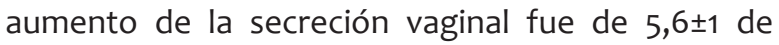
quejas al respeto. Al comparar las medias de las quejas por el aumento de secreción vaginal en los grupos se encuentra diferencia significativa $(p<0,05)$. (Ver Tabla 1)

Tabla 1. Promedio de quejas de aumento en la secreción vaginal

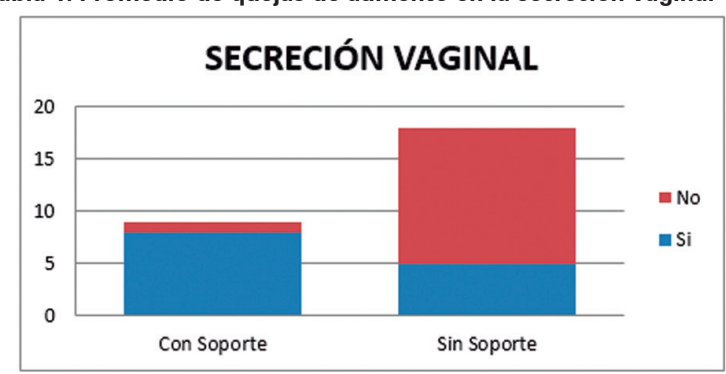

Fuente: Autores.

Respecto a la aparición de erosión y úlceras vaginales, en el grupo manejado con pesario con soporte tuvo una media de erosión y úlceras vaginales de tamaño de $2,47 \mathrm{~mm}(0,5-5,0 \mathrm{~mm})$ en cuatro pacientes (44,4\%), y el grupo con pesario sin soporte de tamaño de $1,43 \mathrm{~mm}$ (0-5,0 mm) en cuatro pacientes (22.2\%). Al comparar las medias es evidente una diferencia significativa en cuanto al tamaño de la lesión $(p=0,045)$. El grupo con pesario sin soporte tuvo una lesión significativamente menor. Se suspendió temporalmente el uso del pesario, durante un tiempo de uno a tres meses, hasta que sanaron las lesiones ulcerosas (Ver Tabla 2).

La impactación en vagina, una complicación relativamente frecuente entre usuarias del pesario, se presentó en cuatro pacientes $(22,2 \%)$ del grupo de las que usaron pesario sin soporte y en dos pacientes $(22,2 \%)$ del grupo de pesario con soporte. No hubo relación entre las variables tipo de pesario e impactación en vagina $(p=0,99)$.

La presencia de atipias citológicas (ASC-US) se presentó en una paciente manejada con pesario con soporte, mientras en las manejadas con pesario sin 
soporte, no se presentaron atipias citológicas. Sin embargo, no hay relación significativa entre los dos tipos de pacientes usuarias de pesarios y la presencia de atipias citológicas, puesto que el proceso inflamatorio crónico que generan ambos es muy similar $(p=0,471)$.

Tabla 2. Erosión y úlceras vaginales promedio de acuerdo al tipo de pesario

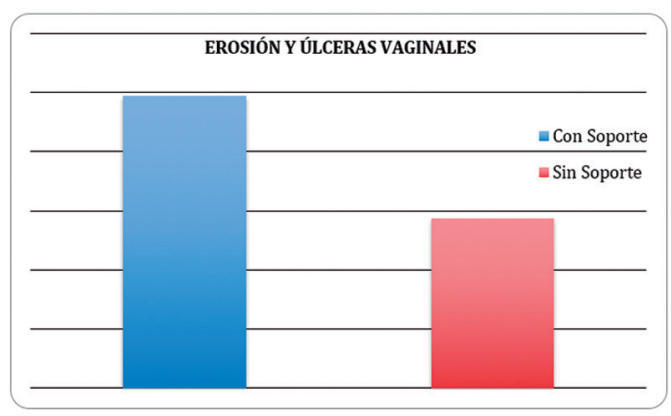

Fuente: Autores.

En relación a la hidronefrosis, se presentó un solo evento en el grupo de usuarias del pesario con soporte y ningún evento en el grupo de usuarias del pesario sin soporte, sin establecerse relación estadísticamente significativa, puesto que el mecanismo de soporte es similar $(p=0,471)$.

De las complicaciones intestinales, se presentó en dos pacientes (22,2\%) del grupo de usuarias del pesario con soporte y en ninguna usuaria del pesario sin soporte. Dicha complicación consistió en retención fecal, en ambas pacientes. No se observó alguna relación entre el tipo de pesario y las complicaciones intestinales, valorada mediante chi cuadrado ( $p=0,299)$, en el sentido de que ninguno de los dos presentaba predominancia sobre el otro. Finalmente, no se encontró relación de mortalidad en los grupos estudiados.

\section{Discusión}

La consulta por el prolapso genital completo, es un evento frecuente en la atención de los servicios de uroginecología y su manejo no siempre es quirúrgico, ya que ofrece alternativas conservadoras como el uso de pesarios, no obstante, representa un reto para el especialista y su equipo de trabajo; en la clínica La Sagrada Familia, el manejo conservador del prolapso, como alternativa no quirúrgica, ha revolucionado su manejo, ofreciendo todos sus beneficios desde hace más de tres años y afortunadamente hasta la fecha, los resultados reportados en el servicio son aceptables.
El presente estudio compara la aparición de complicaciones asociadas al uso de pesario con soporte y sin soporte en el tratamiento del prolapso genital completo. A la fecha no se encuentran en la literatura médica estudios sobre este tema. En este estudio, el prolapso genital completo se presentó con mayor frecuencia en mujeres de edad avanzada, representando alrededor del 59\% del total de pacientes, también se evidenció una tendencia al uso de pesario con soporte en pacientes caucásicas, pero sin encontrarse asociación estadísticamente significativa entre grupos y raza, a pesar de que las pacientes caucásicas tienen mayor tendencia a presentar prolapso genital.

En el grupo de usuarias de pesario con soporte la aparición de erosiones fue en promedio de tamaño de 2,47 mm, siendo significativamente mayor al grupo de usuarias de pesario sin soporte que tuvo una media en el tamaño de la erosión de 1,43 mm ( $p$ $<0,045)$. Lo que se puede explicar por la inhabilidad para ponérselo o quitárselo de parte de las usuarias, al tratarse de mujeres de más de 70 años con patologías severas y funcionalmente incapacitantes; las que usualmente requieren de la ayuda de un tercero para la inserción del pesario.

En síntesis en el adecuado uso del pesario es importante el autocuidado; las pacientes deben quitárselo, limpiarlo y dejarlo fuera de noche y para las relaciones sexuales. Como recomendación para las pacientes, está el insertar el pesario por la mañana y retirarlo en la noche al acostarse, al menos en la primera semana o el primer mes, mientras se familiariza con su uso, sin embargo, en la mujer mucho mayor no siempre es posible, por lo que se sugiere control médico cada uno a tres meses para el seguimiento a largo plazo, mientras que con el autocuidado, el control sería cada seis meses. Es recomendable retirarlo cada noche o al menos cada semana, lavarlo, secarlo y colocárselo de nuevo en la mañana, tal y como lo sugieren algunos autores ${ }^{52}$.

\section{CONCLUSIONES}

El aumento de la secreción vaginal es significativamente menor en pacientes usuarias de pesarios sin soporte en comparación con usuarias de pesarios con soporte, para corrección quirúrgica del prolapso genital completo, además que facilita la actividad sexual sin la necesidad de ser retirado para su adecuada práctica a la hora de la penetración vaginal. 
La aparición de erosión y úlceras vaginales es significativamente menor en pacientes usuarias de pesarios sin soporte en comparación con las pacientes usuarias de pesarios con soporte, para corrección quirúrgica del prolapso genital completo, además de que el tamaño de las úlceras en las usuarias de pesarios sin soporte es menor que el de las usuarias de pesarios con soporte. No hay diferencia significativa del grupo de usuarias de pesarios sin soporte respecto al grupo de usuarias de pesarios con soporte en cuanto a impactación en vagina, fístulas vesicovaginal y rectovaginal, atipias citológicas, incarceración del pesario, hidronefrosis, infección vaginal y complicaciones intestinales.

El uso de pesarios intravaginales en el manejo conservador del prolapso genital completo es posible, y aunque está asociado a complicaciones, estas no son severas. El tratamiento conservador con el uso de pesarios resulta ser económico, de bajo riesgo y eficaz, en el manejo de prolapso genital completo; y del adecuado uso, así como de la educación de las usuarias en el autocuidado, el uso de estrógenos tópicos aunado al prudente seguimiento, dependerá que el pesario sea una óptima solución en el manejo del prolapso genital completo.

\section{FinANCIAMIENTO}

La financiación del estudio estuvo a cargo del autor.

\section{Conflicto de InTERÉs}

El autor confirma no tener algún conflicto de interés.

\section{Agradecimientos}

Al personal de archivos de la clínica La Sagrada Familia por el apoyo durante la recolección de los datos.

\section{Referencias Bibliográficas}

1. ACOG practice bulletin. Pelvic organ prolapse. Obsstet Gynecol 2007;110(3):717-29.

2. Nygaard I, Bradley C, Brandt D; Women`s Health Initiative. Pelvic organ prolapse in older women: Prevalence and risk factors. Obstet Gynecol. 2004;104:489-97.

3. Echavarría LG. Fijación sacroespinosa en el manejo del prolapso de cúpula vaginal y hernia pélvica: diez años de experiencia (1992-2002). Rev Colomb Obstet Ginecol 2004;55:30-4.

4. Bump RC, mattiasson a, Bo K, Brubaker LP, DeLancey JO, Klarskov $\mathrm{P}$ et al. The standardization of terminology of female pelvic organ prolapse and pelvic floor dysfunction. am J Obstet Gynecol 1996;175:10-7.

5. Espitia De La Hoz FJ. Factores de riesgo asociados con prolapso genital femenino: estudio de casos y controles. Rev Urol Colomb. 2015;24(1):12-8.

6. Hendrix SL, Clark A, Nygaard I, Aragaki A, Barnabei V, McTiernan A. Pelvic organ prolapse in the Women's Health Initiative: Gravity and gravidity. Am J Obstet Gynecol. 2002;186:1160-6.

7. Clark AL, Gregory R, Smith VJ, Edwards R. Epidemiologic evaluation of reoperation for surgically treated pelvic organ prolapse and urinary incontinence. Am J Obstet Gynecol 2003; 189: 1261-7.

8. Olsen AL, Smith VJ, Bergstrom JO, Colling JC, Clark AL. Epidemiology of surgically managed pelvic organ prolapse and urinary incontinence. Obstet Gynecol. 1997;89(4):501-6.

9. Samuellson E C, Victor FT A, Tibblin G, Svardsudd K F. Signs of genital prolapsed in a Swedish population of women 20 to 59 years of age and possible related factors. Am J Obstet Gynecol. 1999;180:229-305.

10. Jelovsek JE, Maher C, Barber MD. Pelvic organ prolapse. Lancet 2007; 369: 1027-38.

11. Maher C, Feiner B, Baessler K, Schmid C. Surgical management of pelvic organ prolapse in women. Cochrane Database Syst Rev. 2013 (4):CD004014.

12. Elneil S. Complex pelvic floor failure and associated problems. Best Pract Res Clin Gastroenterol. 2009;23:555-73.

13. Bump RC, Norton PA: Datos epidemiológicos y evolución natural de la disfunción del piso pelviano. Obstet Ginecol, 1998; 4:681.

14. Swift S. Current opinion on the classification and definition of genital tract prolapse. Curr Opin Obstet Gynecol 2002; 14: 503-7.

15. Mant J, Painter R, Vessey M. Epidemiology of genital prolapse: observations from the Oxford Family Planning Association Study. Br J Obstet Gynaecol 1997; 104: 579-85.

16. Klein MC, Gauthier RJ, Robbins JM, Kaczorowski J, Jorgensen SH, Franco ED, y cols. Relationship of episiotomy to perineal trauma and morbidity, sexual disfunction and pelvic floor relaxation. Am J Obstet Gynecol 1994; 171 (3): 591-9.

17. Bump RC, McClish DK. Cigarette smoking and urinary incontinence in woman. Am J Obstet Gynecol 1992; 167(5): 1213-8.

18. Carley ME, Schaffer JI. Urinary incontinence and pelvic organ prolapse in women with marfan or Ehlers Danlos syndrome. am J Obstet Gynecol 2000;182:1021-3.

19. Bradley C, Zimmerman M, Wang Q, et al. Vaginal descent and pelvic floor symptoms in postmenopausal women. A longitudinal study. Obstet Gynecol 2008; 111(5): 1148-53.

20. Kahn M, Breitkopf C, Valley M, et al. Pelvic organ support study (POSST) and bowel symptoms: straining at stool is associated with perineal and anterior vaginal descent in a general gynecology population. Am J Obstet Gynecol 2005; 192: 1516-22.

21. Baden WF, Walker T. Fundamentals, symptoms and classification. In: Baden WF, Walker T, editors. Surgical repair of vaginal defects. Philadelphia (PA): JB Lippincott; 1992.p 14.

22. Bump RC, Mattiasson A, Bo K, et al. The standardization of terminology of female pelvic oragn prolapse and pelvic floor dysfunction. Am J Obstet Gynecol 1996; 175: 10-7.

23. Bernard T. Haylen \& Dirk de Ridder, Robert M. Freeman, et al. An International Urogynecological Association (IUGA)/International Continence Society (ICS) joint report on the terminology for female pelvic floor dysfunction. Int Urogynecol J 2010; 21: 5-26.

24. Vierhout ME, Stoutjesdijk J, Spruijt J. A comparison of preoperative and intraoperative evaluation of patients undergoing pelvic reconstructive surgery for pelvic organ prolapse using the Pelvic Organ Prolapse Quantification System. Int Urogynecol J Pelvic Floor Dysfunct 2006; 17:46.

25. Peschers UM, Schaes GN, DeLancey JO, Schuessler B. Levator ani function before and after Childbirth. Br J Obstet Gynecol 1997; 104(9): 1004-8.

26. Diwan A, Rardin CR, Coolí N. Uterine preservation during surgery for uterovaginal prolapse: a review. Int Urogynecol J Pelvic Floor Dysfunct. 2004;15(4): 286-92.

27. Christopher Maher, Kaven Baessler , Cathryn MA Glazener, Elisabeth J Adams, Suzanne Hagen. Surgical management of pelvic organ prolapse in women. Cochrane Database of Systematic Reviews, Issue 2, 2009.

28. Maher C, Baessler K, Glazener CM, Adams EJ, Hagen S. Surgical management of pelvic organ prolapse in women. Cochrane Database Syst Rev 2007;(3): CD004014.

29. Trowbridge Er, fenner DE. Conservative management of pelvic 
organ prolapse. Clin Obstet Gynecol 2005;48:668-81.

30. Oliver R, Thakar R, Sultan AH. The history and usage of the vaginal pessary: a review. Eur J Obstet Gynecol Reprod Biol. 2011;156:125-30.

31. Fernando RJ, Thakar R, Sultan, et al. Effect of vaginal pessaries on symptoms associated with organ prolapse. Obstet Gynecol 2006; 108(1):93-9.

32. Hanson LA, Schultz JA, Flood CG, et al. Vaginal pessaries in managing women with pelvis organ prolapse and urinary incontinente: patient characteristics and factors contributing to su cess. Int Urogynecol J Pelvic Floor Dysfunct 2006;17(2):155-9.

33. Young JB, Selby PL, Peacock M, Brownjohn AM: Uterine prolapse and urinary tract obstruction. Br Med J 1984; 289: 41-2.

34. Atnip S, O’Dell K. Vaginal support pessaries: indications for use and fitting strategies. Urol Nurs 2012;32:114-24.

35. Lazarou K, Wang A, LaCombe J, et al. Pessary use in advanced pelvic organ prolapse. Int Urogynecol J Pelvic Floor Dysfunct 2006;17(2): 160-4.

36. Gregoir W, Schulman CC, Chantrie M: Ureteric obstruction associated with uterine prolapse. Eur Urol 1976; 2: 29-33.

37. Gleason JL, et al. (2012). Pelvic organ prolapse. In JS Berek, ed., Berek and Novak's Gynecology, 15th ed., pp. 906-939. Philadelphia: Lippincott Williams and Wilkins.

38. Bai SW, Yoon BS, Kwon JY, et al. Survey of the characteristics and satisfaction degree of the patients using a pessary. Int Urogynecol J Pelvic Floor Dysfunct 2005;16(3):182-6.

39. Alexander JL, Rustom R, Bone JM: Acute renal failure from complete uterine prolapse: role of polycystic kidney disease. Clin Nephrol 60: 143-145, 2003.

40. Lamers BH, Broekman BM, Milani AL. Pessary treatment for pelvic organprolapse and health-related quality of life: a review. Int Urogynecol J 2011;22:637-44.

41. Bash K. Review of vaginal pessaries. Obstet Gynecol Surv 2000;55:455-60.
42. Guidelines for the use of support pessaries in the management of pelvic organ prolapse. Adelaide: International Centre for Allied Health Evidence; 2012.

43. Brincat C, Kenton K, Pat Fitzgerald M, Brubaker L. Sexual activity predicts continued pessary use. Am J Obstet Gynecol 2004;191(1):198-200

44. Matsubara S, Ohki Y. Can a ring pessary have a lasting effect to reverse uterine prolapse even alter its renoval? J Obstet Gynecol Res. 2010;36:459-61.

45. Hagen S, Stark D, Maher C, Adams E. Conservative management of pelvic organ prolapsed in women. Cochrane Database Syst Rev 2006 Oct 18; (4): CD003882.

46. Clemons JL, Aguilar VC, Tillinghast TA, et al. Patient satisfaction and changes in prolapse and urinary symptoms in women who were fitted successfully with a pessary for pelvic organ prolapse. Am J Obstet Gynecol 2004; 190(4):1025-9.

47. Rousseau J, Fenton J, Mathieu G, Taleb M. Les cancers vaginaux primitives de l`radulte. A propos de 72 cas traités à la Fondation Curie de 1956 à 1968. Bull Cancer. 1977;64:429-42.

48. Picurelli L, Lopez-Olmos J, Sendra A, Tramoyeres A. Fistula vesicovaginal por cuerpo extra $\sim$ no en vagina. Actas Urol Esp. 1997;21:511-2

49. Powers K, Grigorescu B, Lazarou G, Greston WM, Weber Th. Neglected pessary causing a rectovaginal fistula. A case report. J.Reprod Med. 2008;53:235-7.

50. Stephan WB, Zaaijhan J, du T. Retention of a vaginal ring pessary in a postmenopausal patient. SAMJ. 2007;97:552.

51. Atnip ShD. Uso de pesarios y tratamiento del prolapso de los órganos pélvicos, en Clínicas Obstétricas y Ginecológicas de Norteamérica. En: Schaffer JI, editor. Medicina pélvica y Cirugía reconstructiva en la mujer, 36. Saunders, 2010: Elsevier Masson; 2009. p. 541-63.

52. Hughes D, Schaffer JI. Prolapso de órganos pélvicos. Tratamiento no quirúrgico. En: WILLIAMS Ginecología, de Schorge, Schaffer, Halverson, Hoffman, Bradchaw y Cunningham. 1a ed. 2009, cap 24, p. 545-9. 